\title{
Novel Approach for Determination of Entropy and Compactivity of Granular Matter in Tube
}

\author{
Y. ÖZ* \\ Turkish Aerospace, $R \& \mathcal{G}$ Directorate, Department of Research and Technology Centers, \\ 06980 Kahramankazan, Ankara, Turkey
}

Received: 19.06.2021 \& Accepted: 28.10.2021

Doi: 10.12693/APhysPolA.140.389

*e-mail: yahya@unam.bilkent.edu.tr

\begin{abstract}
Granular matter in a tube is studied in this work. For this purpose, we consider identical disks confined in a tube that has one open and one closed end whereby all surfaces are assumed to be hard and frictionless. Thus, a one-dimensional system is considered. Jamming occurs due to the local pressure in a uniform gravitational field parallel to the tube and rotation. The framework of the configurational statistics with a generalized Pauli principle is used, in which jammed microstates are encoded in particle configurations while macrostates are produced by uniform arbitrary agitations and described by entropy and volume. Moreover, mass density, compactivity and population correlations of quasiparticles are studied analytically.
\end{abstract}

topics: entropy, granular matter, jamming, quasiparticles

\section{Introduction}

Granular matter consists of particles that are so large that thermal fluctuations do not play a significant role in the system. The macroscopic properties of the system result from the microscopic structure of particles, interactions between them and the interactions of particles with external fields. Thus, in this context, thermal properties are overwritten by more macroscopic dynamics. Nonetheless, many studies do not differentiated between systems where there is no long-range order, and consider the granular matter as an area of a larger phase diagram [1-8]. This turns out to be a drawback as thermal interactions usually play a role in such systems.

How the entropy behaves in general granular systems is quite difficult to predict because these systems are not in thermal equilibrium $[9,10]$. Within this regard, in the last decades, a large number of studies and theories on the granular matter or more general systems that are not in thermal equilibrium have been published [11-18] in combination with numerical analyses [19-37].

There is also a whole range of studies on statistical mechanics with only hard-core repulsion as an introduction to granular matter [38-47]. All insignificant properties of grains are neglected in these studies. Note that in this context the energy $k_{\mathrm{B}} T$ is typically compared to the gravitational potential energy $m g \sigma$, where $\sigma$ is the diameter of the considered particle and $g$ is the gravitational field. As a result, in suspension the granular regime is differentiated from the colloidal regime. In this context, gravity and centrifuge [48-51] were used to determine the nature of jammed states. Furthermore, the critical singularities and ordering tendencies were studied based on the entropy of macrostates.

Complementary, the idea of the generalized Pauli principle which is a sound methodology and indisputable because of its main root [52-56], is used in this paper to study jamming due to gravitation and centrifuge. In our model, grains are represented by identical hard and frictionless disks confined in a thin tube, such that two consecutive disks in the interior can take one of two possible configurations, (i) either one vertically above the other, both on the same side of the tube (low density), or (ii) obliquely, touching opposite sides (close packing). The system is subjected to a uniform gravitational field $m g$ along the main axis of the tube and rotated around this axis. According to generally accepted definitions of granular matter, grains are treated as macroscopic objects, large enough to neglect Brownian motions.

The novelty of this approach consists in the application of an important generalization of the Pauli exclusion principle in quantum mechanics [57] by Haldane [58]. In this context, and in cooperation 
with $\mathrm{Wu}$ [59], the statistical properties of the so called quasiparticles in condensed matter physics of low-dimensional systems were extended to the thermodynamics of a system of several species of identical quasiparticles. The methodology is applied to a purely classical model of macroscopic disks in a tube. This task is performed just by a handy association of classical variables with quantum mechanical notions. The statistical interaction between quasiparticles is encoded by characterizing how the position of one quasiparticle changes the distribution options for different quasiparticles. The analyzed sets of quasiparticles prove that granular matter can be examined with this approach since the obtained entropy matches the wellknown results. Characteristics of profiles like volume and mass density are determined exactly. The results show that the balance of forces in combination with the potential energies affect the nature of jamming.

\section{Methodology}

In the generalized Pauli principle [58], the dimension of a Hilbert space $\operatorname{dim} \mathcal{H}_{m}=d_{m}$ changes when quasiparticles are added and the system under consideration does not change otherwise. The statistical interaction coefficients $g_{m n}$ are therefore defined by a differential relation. The equation for $d_{m}$ with statistical capacity constants $A_{m}$ is given by

$$
d_{m}=Y_{m}+g_{m m}
$$

where

$$
Y_{m}=A_{m}-\sum_{n=1}^{M} g_{m n} N_{n}
$$

and $N_{m}$ is the number of quasiparticles of species $m$, $M$ is the number of species. The thermodynamic limit for extensive dimensions $d_{m}$ exists when the statistical interaction coefficients are $g_{m n} \in \mathbb{Q}$. The multiplicity expression for the number of manybody states can be written as

$$
W=n_{\mathrm{pv}} \prod_{n=1}^{M}\left(\begin{array}{c}
d_{m}+N_{m}-1 \\
N_{m}
\end{array}\right),
$$

where $n_{\mathrm{pv}}$ is the multiplicity of the pseudo-vacuum. If there is no dynamic interaction between quasiparticles with energies $\epsilon_{m}$ relative to the pseudovacuum, the total energy of a many-body state given for fixed particle numbers $\left\{N_{m}\right\}$ is

$$
E=E_{\mathrm{pv}}+\sum_{m=1}^{M} N_{m} \epsilon_{m}
$$

where $E_{\mathrm{pv}}$ is the pseudo-vacuum energy.

The analysis of the statistical mechanic of this approach is well-known $[59,60]$. The grand canonical partition function is given by

$$
Z=\prod_{m=1}^{M}\left(1+w_{m}^{-1}\right)^{A_{m}}
$$

where the real and positive quantities $w_{m}$ are given by coupled nonlinear algebraic equations. Namely,

$$
\frac{\epsilon_{m}}{T_{k}}=\ln \left(1+w_{m}\right)-\sum_{n=1}^{M} g_{n m} \ln \left(1+\frac{1}{w_{n}}\right) .
$$

The use of these quantities allows the average numbers of particles to be determined with

$$
A_{m}=w_{m}\left\langle N_{m}\right\rangle+\sum_{n=1}^{M} g_{m n}\left\langle N_{m}\right\rangle .
$$

As the nomenclature, one uses

- compacts (particles without hosting capability that exist side by side in a pseudovacuum),

- hosts (particles with hosting capability that exist side by side in a pseudo-vacuum),

- tags (hosted particles that leave place for other tags without hosting capability),

- caps (hosted particles that leave no place for other caps without hosting capability),

- hybrids (hosted particles with hosting capability),

Thus, in the following compact notation,

$$
\begin{aligned}
& |A\rangle=\left(A_{1}, \ldots, A_{M}\right)^{\mathrm{T}}, \\
& |\epsilon\rangle=\left(\epsilon_{1}, \ldots, \epsilon_{M}\right)^{\mathrm{T}}, \\
& |w\rangle=\left(w_{1}, \ldots, w_{M}\right)^{\mathrm{T}}, \\
& |N\rangle=\left(\left\langle N_{1}\right\rangle, \ldots,\left\langle N_{M}\right\rangle\right)^{\mathrm{T}},
\end{aligned}
$$

and

$$
\mathbf{g}=\left(g_{m n}\right), \quad \mathbf{G}=\mathbf{g}+\left(\delta_{m n} w_{n}\right),
$$

are used. Now, (4) and (7) can be rewritten as

$$
E=E_{\mathrm{pv}}+\langle N \mid \epsilon\rangle, \quad|N\rangle=\mathbf{G}^{-1}|A\rangle,
$$

where $\mathbf{G}$ is assumed to be invertible. The entropy $S=k_{\mathrm{B}} \ln (W)$ is given by

$$
\begin{aligned}
S & =k_{\mathrm{B}} \sum_{m=1}^{M}\left[\left(N_{m}+Y_{m}\right) \ln \left(N_{m}+Y_{m}\right)\right. \\
& \left.-N_{m} \ln \left(N_{m}\right)-Y_{m} \ln \left(Y_{m}\right)\right] .
\end{aligned}
$$

\section{Model}

The model system for studying jamming is a narrow tube of height $H$. The hard, friction-free disks with mass $\mu$ compressed therein are combined into geometric units, for example, two successive disks on the same tube wall. These units have a modeldependent energy $\epsilon_{m}$ and are interpreted as quasiparticles. This enables us to use the methodology described in Sect. 2. The tube is so narrow that $1<\frac{H}{\sigma}<1+\frac{\sqrt{3}}{2}$ applies, where $\sigma$ is the disk diameter. Such structure implies that only four different disk configurations can exist. Each disk touches one wall and two neighbouring disks. The only exceptions are the two outermost disks. Jamming occurs when each disk has three contact points that do not lie on the same semicircle. 


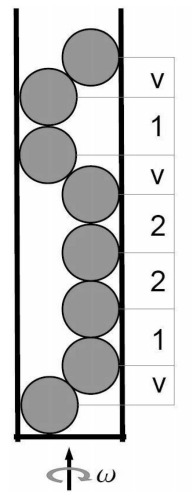

$$
\mathrm{g,z}
$$

Fig. 1. Depiction of both quasiparticles in the tube. The pseudo-vacuum is pointed out with the letter $v$, and $\omega$ characterizes the tube spinning about its axis.

In the presented model, the jammed states are described by the configurations of quasiparticles. The disks themselves are defined as microstates while the macrostates are the merging of disks into quasiparticles. The pseudo-vacuum from which the quasiparticles are excited is chosen as an alternating sequence of one disk on one side and the other on the opposite side of the tube. This pseudo-vacuum is obviously two-fold degenerate, hence $n_{\mathrm{pv}}=2$. The configuration of the model in which the jammed microstates are countable and each has a well defined volume, quasiparticles and pseudo-vacuum are shown in Fig. 1. Quasiparticles populations completely describe the jammed macrostate. Note that $1+\frac{\sqrt{3}}{2}<\frac{H}{\sigma}<2$ leads to 32 different disk configurations while for $\frac{H}{\sigma}>2$ the disks are able to pass each other resulting in a nonlocal requirement for jamming.

The scaled rotation and position are given by $\hat{\omega}^{2}=\mu d \sigma \omega^{2} /\left(4 T_{k}\right)$ and $\hat{z}=\sigma g z / T_{k}$, respectively with $d=\frac{1}{2}(H-\sigma)$, and $T_{k}$ is the parameter for the strength of uniform arbitrary agitations. It plays a major role in the mechanical stability of disk configurations with more than two consecutive disks.

The set of quasiparticles consists of one host and one tag with volume $\sigma$ while the volume of the pseudo-vaccum part is $\sqrt{\frac{H}{\sigma}\left(2-\frac{H}{\sigma}\right)}$. Generating a quasiparticle increases the energy, i.e.,

$$
\begin{aligned}
& \mathbf{g}=\left(\begin{array}{cc}
2 & 1 \\
-1 & 0
\end{array}\right), \quad|A\rangle=\left(\begin{array}{c}
N \\
0
\end{array}\right), \\
& |\epsilon\rangle=\sigma\left(1-\sqrt{\frac{H}{\sigma}\left(2-\frac{H}{\sigma} s\right)}\right) p(z)\left(\begin{array}{l}
1 \\
1
\end{array}\right),
\end{aligned}
$$

where $p(z)$ is the local pressure and $N$ is the number of disks. The dimensionless version of the local pressure is given by $\hat{p}=\sigma\left(1-\sqrt{\frac{H}{\sigma}\left(2-\frac{H}{\sigma}\right)}\right) p(z) / T_{k}$. Hence, the description of the jammed macrostates is performed by the two independent variables pressure $\hat{p}$ and rotation $\hat{\omega}$.

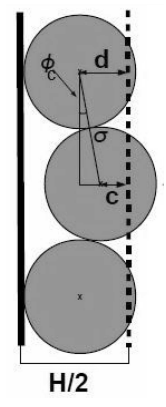

Fig. 2. Depiction of a three disk configuration. The centrifugal force $F_{\omega}=\mu \omega^{2} c$ stabilizes while contact forces $\frac{p(z)}{\cos \phi_{c}}$ destabilize the configuration. The forces are in balance for $c=\frac{d \hat{p}}{\hat{\omega}^{2}} \tan \phi_{c}$.

The energies $|\epsilon\rangle$ of the quasiparticles do not depend on $\hat{\omega}$. However, it is obvious that small rotations $\hat{\omega} \ll 1$ should lead to unstable tags. For the description of this effect three disks are considered. The first assumption is that the disks above and below the middle disk always touch the wall. The second assumption is that the pressure $p(z)$ above and below the middle disk is approximately the same. The configuration is depicted in Fig. 2.

These assumptions generate the function $R_{\omega}=$ $1-\frac{c}{d}=1 /\left(1+\frac{d}{\sigma} \frac{\hat{p}}{\hat{\omega}^{2}}\right)$ which specifies how many tags overcome a reorganization of the system due to mechanical instabilities.

Note that, in principle, there is an alternative procedure for describing the mechanical instabilities. Unlike $R_{\omega}$, a chemical potential $\psi(\hat{\omega})$ can be introduced such that $\epsilon_{2} \rightarrow \epsilon_{2}+\psi(\hat{\omega})$. However, the numerical studies show that in the presented model both populations of quasiparticles simultaneously tend to zero for small $\frac{\psi(\hat{\omega})}{\hat{p}}$. This possible modification of the model is not investigated further since the population density of the host quasiparticle does not depend on mechanical instabilities.

\section{Results}

The form of (6) and (12) is then

$$
w=w_{1}=w_{2}=\mathrm{e}^{\hat{p}},
$$

and

$$
\mathbf{G}^{-1}=\frac{1}{(1+w)^{2}}\left(\begin{array}{cc}
w & -1 \\
1 & 2+w
\end{array}\right) .
$$

\subsection{Population densities}

Population densities $\bar{N}_{1}=\lim _{N \rightarrow \infty} \frac{\left\langle N_{1}\right\rangle}{N}$ and $\bar{N}_{2}=R_{\omega} \lim _{N \rightarrow \infty} \frac{\left\langle N_{2}\right\rangle}{N}$ are given by

$$
|\bar{N}\rangle=\left(\begin{array}{c}
\bar{N}_{1} \\
\bar{N}_{2}
\end{array}\right)=\frac{1}{(1+w)^{2}}\left(\begin{array}{c}
w \\
R_{\omega}
\end{array}\right),
$$




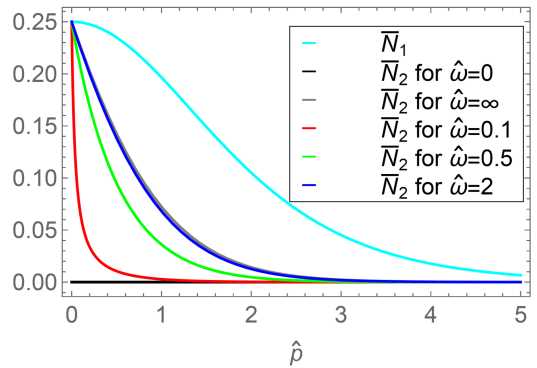

Fig. 3. Course of population densities $\bar{N}_{1}$ and $\bar{N}_{2}$ for growing pressures $\hat{p}$. The results for varying rotations $\hat{\omega}$ and $d / \sigma=\frac{1}{4}$ are shown. The black and gray curves depict the cases for $\hat{\omega}=0$ and $\hat{\omega} \rightarrow \infty$, i.e., $\bar{N}_{2}=0$ and $\bar{N}_{2}=\left(1+\mathrm{e}^{\hat{p}}\right)^{-2}$, respectively. The curves for finite rotations $\hat{\omega}$ lie between these two cases. The blue curve for $\hat{\omega}=2$ lies almost on top of the curve for $\hat{\omega} \rightarrow \infty$.

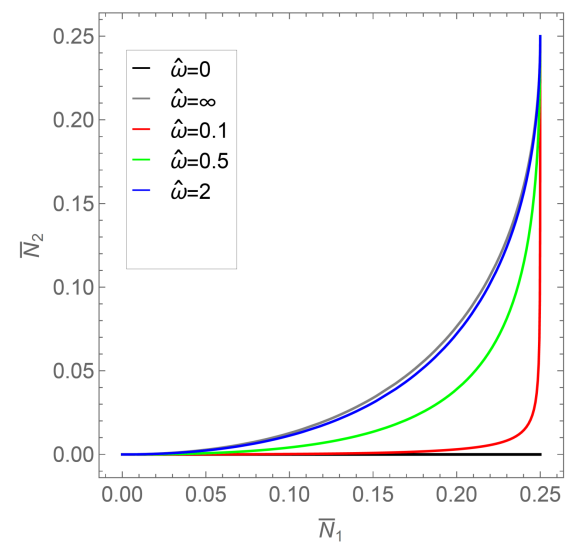

Fig. 4. Depiction of the relation between $\bar{N}_{1}$ and $\bar{N}_{2}$ for $\frac{d}{\sigma}=\frac{1}{4}$. The results obtained for $\hat{\omega}=0.1$, $0.5,1,2, \infty$ become steeper for $\bar{N}_{1} \approx \frac{1}{4}$ with decreasing $\hat{\omega}$.

where $R_{\omega}$ appears due to the mechanical instabilities. For small and large rotations $\hat{\omega}$, we find

$$
\bar{N}_{2}= \begin{cases}\frac{\sigma \hat{\omega}^{2}}{d \hat{p}}\left(1+\mathrm{e}^{\hat{p}}\right)^{-2}+\mathcal{O}\left(\hat{\omega}^{2}\right), & \hat{\omega} \ll 1, \\ \left(1+\mathrm{e}^{\hat{p}}\right)^{-2}+\mathcal{O}\left(\hat{\omega}^{-2}\right), & \hat{\omega} \gg 1 .\end{cases}
$$

The population density $\bar{N}_{2}$ for quasiparticle 2 vanishes for small rotations $\hat{\omega}$. Figure 3 shows the results for the population densities $|\bar{N}\rangle$ for varying rotations $\hat{\omega}$.

Note that $\hat{p} \rightarrow 0$ yields $\hat{z} \rightarrow 0$. For small or large $\hat{p}$, the population densities simplify to

$$
\begin{aligned}
& \bar{N}_{1}= \begin{cases}\frac{1}{4}+\mathcal{O}\left(\hat{p}^{2}\right), & \hat{p} \ll 1, \\
\mathrm{e}^{-\hat{p}}+\mathcal{O}\left(\mathrm{e}^{-2 \hat{p}}\right), & \hat{p} \gg 1,\end{cases} \\
& \bar{N}_{2}= \begin{cases}\frac{1}{4}+\mathcal{O}(\hat{p}), & \hat{p} \ll 1, \\
\frac{\sigma \hat{\omega}^{2}}{d \hat{p}} \mathrm{e}^{-2 \hat{p}}+\mathcal{O}\left(\left(\hat{p} \mathrm{e}^{\hat{p}}\right)^{-2}\right), & \hat{p} \gg 1,\end{cases}
\end{aligned}
$$

which is also observable in Fig. 3.
Due to (19) the presented model has properties adapted to the expected physics, namely, for $\hat{\omega}=0$ (this case) the unstable particle 2 vanishes. The system is dominated by the pseudovacuum and particle 1 . If $\hat{p}=0$ is given additionally, particle 1 and pseudo-vacuum have the same particle energy which leads to an equal weighting by the configuration statistics. Exactly half of the disk configuration is pseudo-vacuum. The other half consists of the state in which the amount of particle 1 is maximized. Hence, a quarter of the total configuration consists of particle 1. Accordingly, $\hat{p}=0$ leads to $\bar{N}_{1}=\frac{1}{4}$. Note that the value $\frac{1}{2}$, which is in principle the highest possible density of particle 1 in Fig. 1, is not reached within this model.

Figure 4 shows how particles 1 and 2 are related. The host-tag relationship of the quasiparticles is observed. If particle 1 occurs more often, it is also more likely to find particle 2 that is attached to it.

\subsection{Volume}

Summation of the population densities leads to the normalized particle volume $\bar{V}=\lim _{N \rightarrow \infty} \frac{V-V_{0}}{N}$ with $V_{0}$ being the volume of the maximally compact configuration, i.e.

$$
\bar{V}=\bar{N}_{1}+\bar{N}_{2}=\frac{R_{\omega}+w}{(1+w)^{2}} .
$$

It reaches its maximum value for $\hat{p}=0$, which is $\frac{1}{4}$ for $\hat{\omega}=0$ due to vanishing tags, and $\frac{1}{2}$ for $\hat{\omega} \neq 0$ while large $\hat{p}$ yields a volume tending to zero that behaves like $\mathrm{e}^{-\hat{p}}+\mathcal{O}\left(\mathrm{e}^{-2 \hat{p}}\right)$. For small or large $\hat{\omega}$ one gets

$$
\bar{V}= \begin{cases}\frac{1}{4 \cosh ^{2}\left(\frac{\hat{p}}{2}\right)}+\mathcal{O}\left(\hat{\omega}^{2}\right), & \hat{\omega} \ll 1, \\ \left(1+\mathrm{e}^{\hat{p}}\right)^{-1}+\mathcal{O}\left(\hat{\omega}^{-2}\right), & \hat{\omega} \gg 1 .\end{cases}
$$

These cases are depicted as black and grey curves in Fig. 5.

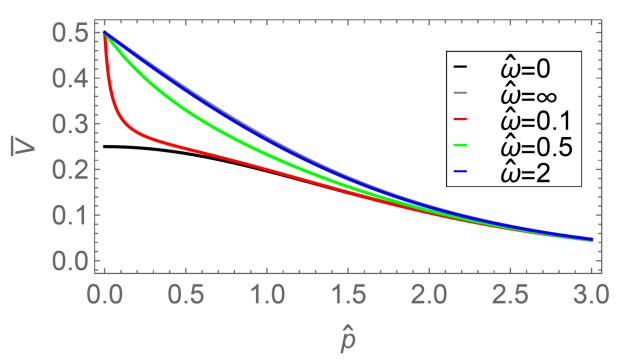

Fig. 5. Volume $\bar{V}$ is given for $\frac{d}{\sigma}=\frac{1}{4}$ and $\hat{\omega}=0$, $0.1,0.5,2, \infty$. The volume shows different behaviour for $\hat{\omega}=0$ and $\hat{\omega} \neq 0$. For $\hat{\omega}=0$ we find $\bar{V}=\frac{1}{4}+$ $\mathcal{O}\left(\hat{p}^{2}\right)$ for small $\hat{p}$ due to vanishing number of tags. In contrast, $\bar{V}=\frac{1}{2}+\mathcal{O}(\hat{p})$ is observable for $\hat{\omega} \neq$ 0 and small $\hat{p}$. Increasing $\hat{\omega}$ leads to an increased volume due to an increasing number of tags. 


\subsection{Mass density}

The mass density of the system is given by

$$
\rho=\frac{\mu}{\sigma(1+\bar{V})}=\frac{\mu}{\sigma} \frac{(1+w)^{2}}{1+R_{\omega}+3 w+w^{2}} .
$$

For small and large rotations $\hat{\omega}$, one gets

$$
\rho= \begin{cases}\frac{4 \mu}{\sigma} \frac{\cosh ^{2}\left(\frac{\hat{p}}{2}\right)}{1+4 \cosh ^{2}\left(\frac{\hat{p}}{2}\right)}+\mathcal{O}\left(\hat{\omega}^{2}\right), & \hat{\omega} \ll 1, \\ \frac{\mu}{\sigma} \frac{1+\mathrm{e}^{\hat{p}}}{2+\mathrm{e}^{\hat{p}}}+\mathcal{O}\left(\hat{\omega}^{-2}\right), & \hat{\omega} \gg 1 .\end{cases}
$$

Large pressures $\hat{p}$ lead to $\frac{\mu}{\sigma}+\mathcal{O}\left(e^{-\hat{p}}\right)$ while pressures $\hat{p}$ result in $\frac{4 \mu}{5 \sigma}+\mathcal{O}\left(\hat{p}^{2}\right)$ for $\hat{\omega}=0$ which is larger than $\frac{2 \mu}{3 \sigma}+\mathcal{O}(\hat{p})$ for $\hat{\omega} \neq 0$ due to an increasing number of tags. This behaviour is shown in Fig. 6 .

\subsection{Packing factor}

The packing factor

$$
\phi=\frac{\pi \sigma^{2}}{4 H^{2}\left(\sqrt{\frac{2 \sigma}{H}-1}+\bar{V}\left(\frac{\sigma}{H}-\sqrt{\frac{2 \sigma}{H}-1}\right)\right)}
$$

simplifies to

$$
\phi=\left\{\begin{array}{l}
\frac{\pi \sigma^{2}(1+\cosh (\hat{p}))}{2 H\left(\sigma+H \sqrt{\frac{2 \sigma}{H}-1}(1+2 \cosh \hat{p})\right)}+\mathcal{O}\left(\hat{\omega}^{2}\right), \\
\hat{\omega} \ll 1, \\
\frac{\pi \sigma^{2}\left(1+\mathrm{e}^{\hat{p}}\right)}{4 H\left(\sigma+H \mathrm{e}^{\hat{p}} \sqrt{\frac{2 \sigma}{H}-1}\right)}+\mathcal{O}\left(\hat{\omega}^{-2}\right), \\
\hat{\omega} \gg 1
\end{array}\right.
$$

for small or large rotations $\hat{\omega}$. Large pressure $\hat{p}$ leads to the maximum packing

$$
\phi=\frac{\pi \sigma^{2}}{4 H^{2} \sqrt{\frac{2 \sigma}{H}-1}}+\mathcal{O}\left(e^{-\hat{p}}\right),
$$

while the minimum packing is given for small pressure $\hat{p}$ by

$$
\phi=\frac{\pi \sigma^{2}}{H\left(\sigma+3 H \sqrt{\frac{2 \sigma}{H}-1}\right)}+\mathcal{O}\left(\hat{p}^{2}\right)
$$

for $\hat{\omega}=0$ or

$$
\phi=\frac{\pi \sigma^{2}}{2 H\left(\sigma+H \sqrt{\frac{2 \sigma}{H}-1}\right)}+\mathcal{O}(\hat{p})
$$

for $\hat{\omega} \neq 0$. The packing factor $\phi$ given by (27) and its corresponding behaviour described by (28)-(31) is given in Fig. 7.

\subsection{Entropy}

The exact entropy per disk $\bar{S}=\lim _{N \rightarrow \infty} \frac{S}{k_{\mathrm{B}} N}$ is straightforwardly obtained from (14). It behaves differently for $\hat{\omega}=0$ and $\hat{\omega} \neq 0$ which can be seen in the result for small rotations $\hat{\omega}$, i.e.,

$$
\begin{aligned}
\bar{S} & =\frac{2 \mathrm{e}^{\hat{p}}}{\left(1+\mathrm{e}^{\hat{p}}\right)^{2}}\left[\frac{1}{2} \ln (2)-\cosh (\hat{p}) \ln (\cosh (\hat{p}))\right. \\
& \left.+\left(\frac{1}{2}+\cosh (\hat{p})\right) \ln \left(\frac{1}{2}+\cosh (\hat{p})\right)\right] \\
& +\mathcal{O}\left(\hat{\omega}^{2}\right)
\end{aligned}
$$

compared to the expression for large rotations $\hat{\omega}$, i.e.,

$$
\begin{aligned}
\bar{S} & =\frac{\mathrm{e}^{\hat{p}}}{\left(1+\mathrm{e}^{\hat{p}}\right)^{2}}\left[\ln (16)+\hat{p}\left(1-\mathrm{e}^{\hat{p}}\right)\right. \\
& \left.+4 \sinh ^{2}\left(\frac{\hat{p}}{2}\right) \ln \left(1+\mathrm{e}^{\hat{p}}\right)+4 \ln \left(\cosh \left(\frac{\hat{p}}{2}\right)\right)\right] \\
& +\mathcal{O}\left(\hat{\omega}^{-2}\right) .
\end{aligned}
$$

Large pressure $\hat{p}$ leads to $\bar{S}=\hat{p} \mathrm{e}^{-\hat{p}}+\mathcal{O}\left(\mathrm{e}^{-\hat{p}}\right)$ while small pressure $\hat{p}$ results in $\bar{S}=\frac{3}{4} \ln (3)-\frac{1}{2} \ln (2)+$ $\mathcal{O}\left(\hat{p}^{2}\right)$ for $\hat{\omega}=0$ and $\bar{S}=\ln (2)+\mathcal{O}\left(\hat{p}^{2}\right)$ for $\hat{\omega} \neq 0$. The entropy $\bar{S}$ depending on the pressure $\hat{p}$ and the packing factor $\phi$ is given in Fig. $8 \mathrm{a}$ and b, respectively. The maximum value is reached at the beginning of the tube since at this point the particle densities $\bar{N}_{1}$ and $\bar{N}_{2}$ or equivalently the volume $\bar{V}$ reach the highest values. Thus, a large number of variations of possible tube configurations is allowed. In turn, for high packing densities $\phi$ or equivalently,

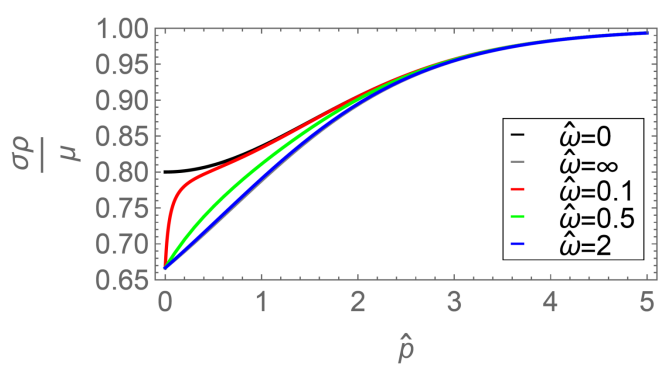

Fig. 6. Density $\rho$ is given for $\frac{d}{\sigma}=\frac{1}{4}$ and $\hat{\omega}=0$, $0.1,0.5,2, \infty$. The mass density shows different behaviour for $\hat{\omega}=0$ and $\hat{\omega} \neq 0$. Increasing $\hat{\omega}$ leads to a decreasing mass density due to an increasing number of tags.

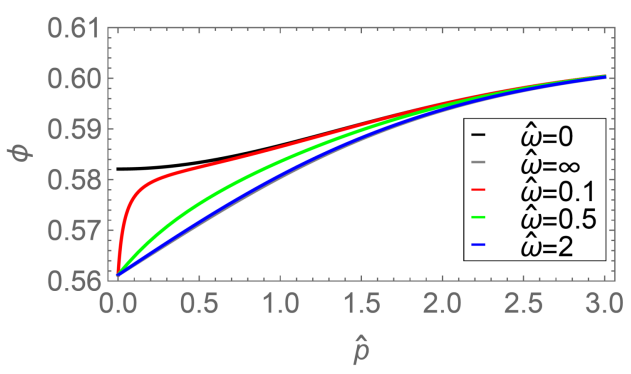

Fig. 7. Packing factor $\phi$ is given for $\frac{d}{\sigma}=\frac{1}{4}$ and $\hat{\omega}=0,0.1,0.5,2, \infty$. The packing factor shows different behaviour for $\hat{\omega}=0$ and $\hat{\omega} \neq 0$. Increasing rotation $\hat{\omega}$ leads to a decreasing packing factor. 

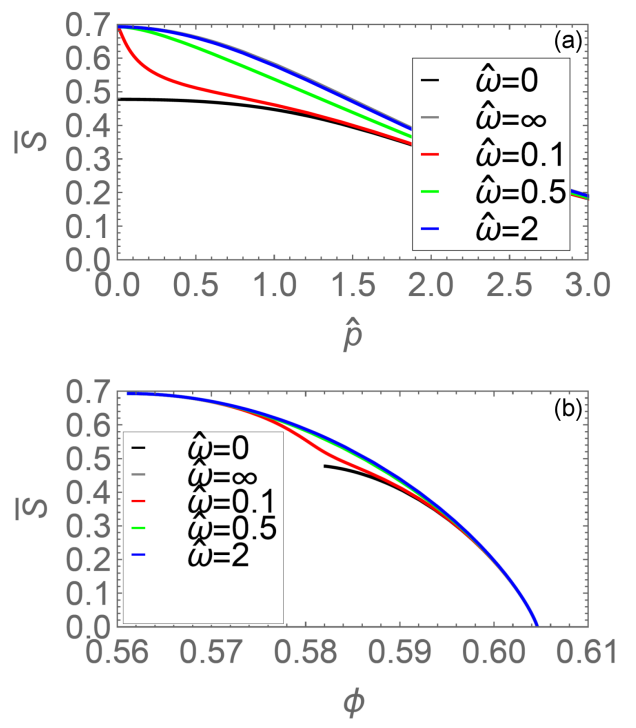

Fig. 8. The entropy $\bar{S}$ is given in dependence of the (a) pressure $\hat{p}$ and (b) packing factor $\phi$ for $\frac{d}{\sigma}=\frac{1}{4}$ and $\hat{\omega}=0,0.1,0.5,2, \infty$. The different $\hat{S}$ behaviour is shown for $\hat{\omega}=0$ and $\hat{\omega} \neq 0$. Increasing $\hat{\omega}$ leads to an increasing entropy. At $\bar{S}=0$ the volume is $\bar{V}=0$ and the system consists entirely of the pseudo-vacuum, i.e., $\bar{N}_{1}=\bar{N}_{2}=0$. At the maximum of the black curve the population densities of the quasiparticles are $\bar{N}_{1}=\frac{1}{4}$ and $\bar{N}_{2}=0$. Hence, at this point a quarter of the system consists of particle 1 while $\frac{3}{4}$ of the system are given by the pseudo-vacuum. The maximum of the entropy lies at $\ln (2)$. Here, half of the system consists of the pseudo-vacuum. However, the other half is splitted evenly into particle 1 and particle 2, i.e., $\bar{N}_{1}=\bar{N}_{2}=\frac{1}{4}$.

when the quasiparticles are completely replaced by the pseudo-vacuum, the entropy $\bar{S}$ vanishes. Remarkably, the entropy shows a similar course as in previous publications [61], even though fundamentally different methods were used in these studies. This confirms, in fact, that the presented approach is justified.

\subsection{Compactivity}

Compactivity, which is the inverse slope of the entropy $\bar{S}$ with respect to the volume $\bar{V}$, is defined by $X=\frac{\partial \bar{V}}{\partial \bar{S}}$ for jammed macrostates depending on only one intensive variable [36]. The compactivity describes the intensity of uniform arbitrary agitations of the configuration.

The entropy $\bar{S}$ depending on volume $\bar{V}$ is a concave function starting at 0 for $\bar{V}=0$ and ending at $\frac{3}{4} \ln (3)-\frac{1}{2} \ln (2)$ or $\ln (2)$ for $\hat{\omega}=0$ or $\hat{\omega} \neq 0$, respectively. Its final slope is zero while its initial slope is infinite for $\hat{\omega} \neq 0$. For $\hat{\omega}=0$, the initial slope is $\left(\ln \left(\frac{4}{3}\right)\right)^{-1}$. Hence, the reciprocal compactivity $X^{-1}$ varies accordingly and it can be simplified as follows

$$
X^{-1}=\left\{\begin{array}{l}
2 \ln \left(\frac{\cosh (\hat{p})\left(1+\mathrm{e}^{\hat{p}}\right)^{2}}{\mathrm{e}^{\hat{p}}(1+\cosh (\hat{p})) \sqrt{1+2 \cosh (\hat{p})}}\right)+\mathcal{O}\left(\hat{\omega}^{2}\right) \\
\quad \hat{\omega} \ll 1 \\
\hat{p}, \quad \\
\quad \hat{\omega} \gg 1
\end{array}\right.
$$

for small and large rotations $\hat{\omega}$. The compactivity shows a $\hat{p}+\mathcal{O}(1)$ behaviour for $\hat{p} \gg 1$ while $\ln \left(\frac{4}{3}\right)+\mathcal{O}\left(\hat{p}^{2}\right)$ or $\left(1+\frac{d}{\sigma \hat{\omega}^{2}}\right) \hat{p}+\mathcal{O}\left(\hat{p}^{2}\right)$ is found for $\hat{p} \ll 1$ and $\hat{\omega}=0$ or $\hat{\omega} \neq 0$, respectively. The reciprocal compactivity $X^{-1}$ diverges for large pressures $\hat{p}$ and its behaviour is depicted in Fig. 9a. Moreover, the dependence on the packing factor and entropy are shown in Fig. 9b and c, respectively. We recognize the well-known tendency of the system to become particularly unstable in perfectly ordered configurations. Specifically, there is a divergence for the maximum of the packing factor given in (29), i.e., when the complete system consists of the pseudo-vacuum. In analogy, the reciprocal compactivity $X^{-1}$ diverges for $\bar{S}=0$.
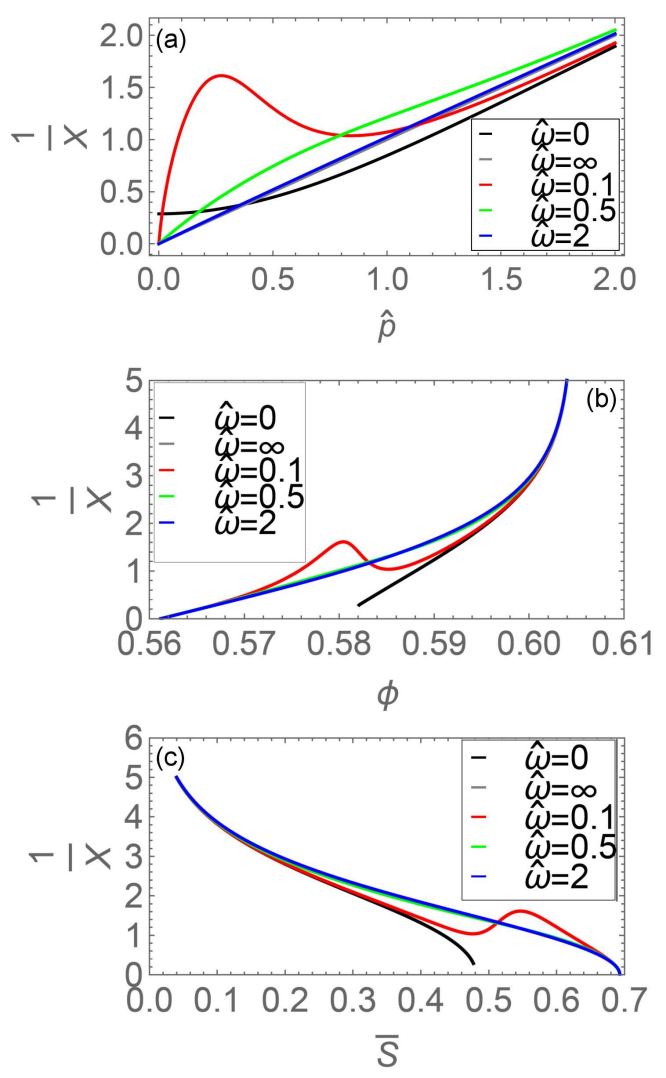

Fig. 9. Reciprocal compactivity $X^{-1}$ in dependence of (a) pressure $\hat{p}$, (b) packing factor $\phi$ and (c) entropy $\bar{S}$ for $\frac{d}{\sigma}=\frac{1}{4}$ and $\hat{\omega}=0,0.1,0.5,2$, $\infty$. For large pressure $\hat{p}$ it behaves linear while it is 0 for $\hat{p}=0$ and $\hat{\omega} \neq 0$. However, $\ln \left(\frac{4}{3}\right)$ is found for $\hat{p}=\hat{\omega}=0$. A transition for $\hat{\omega}=0$ and $\hat{\omega} \neq 0$ is observable. Moreover, $X^{-1}$ diverges for the maximum value of the packing factor $\phi$ and the minimum value of the entropy $\bar{S}=0$. 


\subsection{Population correlations}

Normalized population correlations introduced in [62] and applied to the set of quasiparticles in this study are given as

$$
\rho_{m n}=\frac{w(1+w)}{\sqrt{\bar{N}_{m} \bar{N}_{n}}} \sum_{k=1}^{2} G_{m k}^{-1} G_{n k}^{-1} \bar{N}_{k} .
$$

Note that $\rho_{m n}=\rho_{n m}$ holds. Hence, the three relevant population correlations are expressed as

$$
\begin{aligned}
& \rho_{11}=\frac{\mathrm{e}^{3 \hat{p}}+R_{\omega}}{R_{\omega}\left(1+\mathrm{e}^{\hat{p}}\right)} \sqrt{\bar{N}_{1} \bar{N}_{2}}, \\
& \rho_{12}=\frac{\mathrm{e}^{2 \hat{p}}-R_{\omega}\left(2+\mathrm{e}^{\hat{p}}\right)}{R_{\omega}\left(1+\mathrm{e}^{\hat{p}}\right)} \sqrt{\bar{N}_{1} \bar{N}_{2}}, \\
& \rho_{22}=\frac{\mathrm{e}^{\hat{p}}+R_{\omega}\left(2+\mathrm{e}^{\hat{p}}\right)^{2}}{R_{\omega}\left(1+\mathrm{e}^{\hat{p}}\right)} \sqrt{\bar{N}_{1} \bar{N}_{2}}
\end{aligned}
$$

For small or large rotations $\hat{\omega}$, we find

$$
\begin{aligned}
& \rho_{11}= \begin{cases}\frac{\mathrm{e}^{\frac{7 \hat{p}}{2}}}{\hat{\omega}\left(1+\mathrm{e}^{\hat{p}}\right)^{3}} \sqrt{\frac{d \hat{p}}{\sigma}}+\mathcal{O}(\hat{\omega}), & \hat{\omega} \ll 1, \\
\frac{\mathrm{e}^{\frac{\hat{p}}{2}}\left(1-\mathrm{e}^{\hat{p}}+\mathrm{e}^{2 \hat{p}}\right)}{\left(1+\mathrm{e}^{\hat{p}}\right)^{2}}+\mathcal{O}\left(\hat{\omega}^{-2}\right), & \hat{\omega} \gg 1,\end{cases} \\
& \rho_{12}= \begin{cases}\frac{\mathrm{e}^{\frac{5 \hat{p}}{2}}}{\hat{\omega}\left(1+\mathrm{e}^{\hat{p}}\right)^{3}} \sqrt{\frac{d \hat{p}}{\sigma}}+\mathcal{O}(\hat{\omega}), & \hat{\omega} \ll 1, \\
\frac{\mathrm{e}^{\frac{\hat{p}}{2}}\left(\mathrm{e}^{\hat{p}}-2\right)}{\left(1+\mathrm{e}^{\hat{p}}\right)^{2}}+\mathcal{O}\left(\hat{\omega}^{-2}\right), & \hat{\omega} \gg 1,\end{cases} \\
& \rho_{22}= \begin{cases}\frac{\mathrm{e}^{\frac{3 \hat{p}}{2}}}{\hat{\omega}\left(1+\mathrm{e}^{\hat{p}}\right)^{3}} \sqrt{\frac{d \hat{p}}{\sigma}}+\mathcal{O}(\hat{\omega}), & \hat{\omega} \ll 1, \\
\frac{\mathrm{e}^{\frac{\hat{p}}{2}}\left(4+\mathrm{e}^{\hat{p}}\right)}{\left(1+\mathrm{e}^{\hat{p}}\right)^{2}}+\mathcal{O}\left(\hat{\omega}^{-2}\right), & \hat{\omega} \gg 1 .\end{cases}
\end{aligned}
$$

For small rotations $\hat{\omega}$ and small pressures $\hat{p}$ all population correlations simplify to $\frac{1}{8 \hat{\omega}} \sqrt{\frac{d \hat{p}}{\sigma}}+\mathcal{O}\left(\hat{p}^{\frac{3}{2}} \hat{\omega}^{-1}\right)$ and diverge for $\hat{\omega}=0$. However, small pressures $\hat{p}$ and $\hat{\omega} \neq 0$ leads to $\rho_{11}=\frac{1}{4}+\mathcal{O}(\hat{p}), \rho_{12}=-\frac{1}{4}+\mathcal{O}(\hat{p})$ and $\rho_{22}=\frac{5}{4}+\mathcal{O}(\hat{p})$. Moreover, the population correlations behave for large pressures $\hat{p}$ as

$$
\begin{aligned}
& \rho_{11}=\frac{1}{\hat{\omega}} \sqrt{\frac{d \hat{p}}{\sigma}} \mathrm{e}^{\frac{\hat{p}}{2}}+\mathcal{O}\left(\sqrt{\hat{p}} \mathrm{e}^{-\hat{p} / 2}\right), \\
& \rho_{12}=\frac{1}{\hat{\omega}} \sqrt{\frac{d \hat{p}}{\sigma}} \mathrm{e}^{-\frac{\hat{p}}{2}}+\mathcal{O}\left(\sqrt{\hat{p}} \mathrm{e}^{-3 \hat{p} / 2}\right), \\
& \rho_{22}=\hat{\omega} \sqrt{\frac{\sigma}{d \hat{p}}} \mathrm{e}^{-\frac{\hat{p}}{2}}+\mathcal{O}\left(\sqrt{\hat{p}} \mathrm{e}^{-3 \hat{p} / 2}\right) .
\end{aligned}
$$

The population correlations (36)-(38) and their respective behaviour analytically described by (39)-(44) are depicted in Fig. 10a-c.

The curve of the population correlation $\rho_{11}$ shows that the quasiparticle of species 1 is subjected to strong fluctuations as the pressure $\hat{p}$ increases, which leads to an increasing population correlation $\rho_{11}$. In addition, the population correlation $\rho_{12}$ is zero if

$$
\left(1+\frac{d \hat{p}}{\sigma \hat{\omega}^{2}}\right) \mathrm{e}^{2 \hat{p}}-\mathrm{e}^{\hat{p}}-2=0
$$

holds. For $\hat{\omega} \rightarrow \infty$, we find $\hat{p}=\ln (2)$ as a root. This means that the population correlation changes its
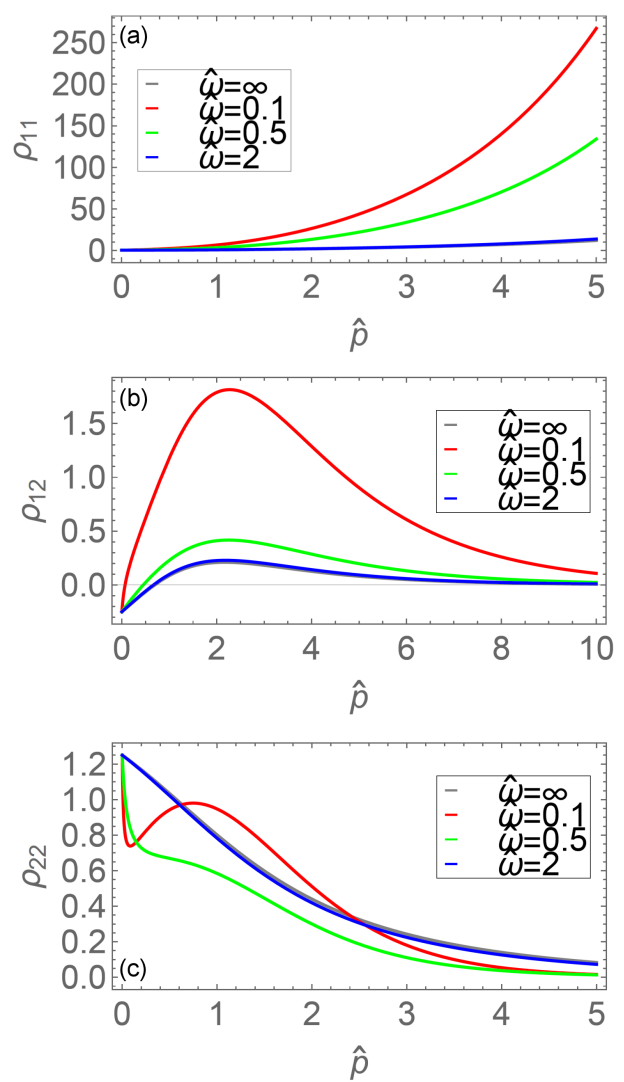

Fig. 10. Population correlations (a) $\rho_{11}$, (b) $\rho_{12}$ and (c) $\rho_{22}$ in dependence of $\hat{p}$ for $\frac{d}{\sigma}=\frac{1}{4}$ and $\hat{\omega}=0.1,0.5,2, \infty$ are shown. The negative correlation in $\rho_{12}$ for small $\hat{p}$ occurs due to the tearing off of longer chains by the insertion of a quasiparticle of species 1 .

sign for sufficiently large pressure $\hat{p}$. At the beginning of the tube, it is initially negative. Afterwards, the population correlation $\rho_{12}$ changes its sign for sufficiently high pressure $\hat{p}$, until it vanishes due to the absence of quasiparticles. As far as the pressure $\hat{p}$ is large, species 2 is stable and relatively frequent. Let us assume that a chain of particles of species 2 exists. A particle of type 1 leads inevitably to the rupture of this chain. The correlation is then partly negative. In the deeper tube region, the quasiparticles become rarer and there are no chains. In this case, particle 2 always benefits over the first species and the correlation is positive. Additionally, when it comes to combinations, there are much more possibilities for quasiparticle 2 to appear in the system if the number of quasiparticles 1 is sufficient. Hence, the population correlation $\rho_{22}$ decreases. Furthermore, the curve for small rotations $\hat{\omega}$ shows a minimum and a maximum due to infinite $\rho_{22}$ for $\hat{\omega}=0$. Small pressure $\hat{p}$ leads to strong positive correlations due to the existence of many quasiparticles of species 2 . If quasiparticle 2 is very rare, it benefits especially strongly from the existence of quasiparticle 1 because it becomes more frequent in combination. 


\subsection{Analyses of limiting cases}

Here, a narrow tube with height $H$ and hard, friction-free disks with diameters $\sigma$ are considered, and $1<\frac{H}{\sigma}<1+\frac{\sqrt{3}}{2}$ holds such that only nearest neighbours interact. The other regimes, i.e., $1+\frac{\sqrt{3}}{2}<\frac{H}{\sigma}<2$ and $\frac{H}{\sigma}>2$ exist as well. The latter requires a nonlocal jamming condition, while the beforementioned leads to 32 different disk arrangements. For these two regimes one need to adjust (15) and (16) with novel sets of quasiparticles. Hence, the limits:

- $\frac{H}{\sigma} \rightarrow 1^{+}$(disk diameter equals container diameter),

- $\frac{H}{\sigma} \rightarrow\left(1+\frac{\sqrt{3}}{2}\right)^{-}$(disk diameter allows only nearest neighbour interactions),

- $\frac{H}{\sigma} \rightarrow\left(1+\frac{\sqrt{3}}{2}\right)^{+}$(disk diameter allows additional interactions),

- $\frac{H}{\sigma} \rightarrow 2^{-}$(two disks fit side by side in the container),

- $\frac{H}{\sigma} \rightarrow 2^{+}$(disks can pass each other)

describe the crossovers between the regimes. Within the scope of this study $\frac{H}{\sigma} \rightarrow 1^{+}$and $\frac{H}{\sigma} \rightarrow\left(1+\frac{\sqrt{3}}{2}\right)^{-}$ can be studied.

On one hand, for disks with diameters equal to the tube height, i.e., $\frac{H}{\sigma} \rightarrow 1^{+}$, the packing factor in (27) leads to

$$
\lim _{\frac{H}{\sigma} \rightarrow 1^{+}} \phi=\frac{\pi}{4} \text {. }
$$

while results for the population densities $\lim _{\frac{H}{\sigma} \rightarrow 1^{+}}|\bar{N}\rangle$, the volume $\lim _{\frac{H}{\sigma} \rightarrow 1^{+}} \bar{V}$, the mass density $\lim _{\frac{H}{\sigma} \rightarrow 1^{+}} \rho$, the entropy $\lim _{\frac{H}{\sigma} \rightarrow 1^{+}} \bar{S}$, the compactivity $\lim _{\frac{H}{\sigma} \rightarrow 1^{+}} X$ and the population correlations $\lim _{\frac{H}{\sigma} \rightarrow 1^{+}} \rho_{m n}(m, n=1,2)$ simplify to expressions given in (19) in addition to (20), (24), (26), (33), (34) and (39)-(41) for infinitely large

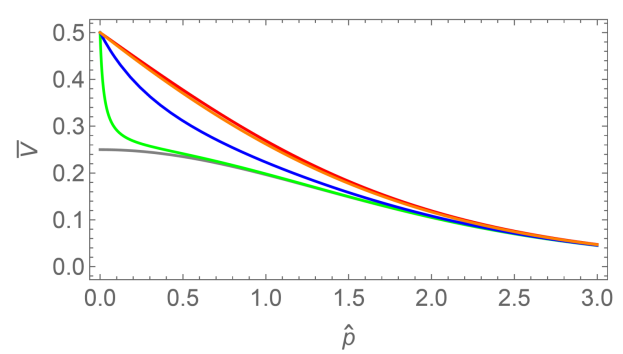

Fig. 11. Volumes $\bar{V}$ for $\frac{H}{\sigma} \rightarrow 1^{+}$(black curve) and $\frac{H}{\sigma} \rightarrow\left(1+\frac{\sqrt{3}}{2}\right)^{-}$with $\hat{\omega}=0$ (gray curve), 0.1 (red curve), 0.5 (green curve), 2 (blue curve), $\infty$ (orange curve) are depicted. Curves for $\frac{H}{\sigma} \rightarrow 1^{+}$and $\omega=\infty$ lie on top of each other while results for $\frac{H}{\sigma} \rightarrow(1+$ $\left.\frac{\sqrt{3}}{2}\right)^{-}$are decreased in comparison to the volume presented in Fig. 5 for $\frac{H}{\sigma}=\frac{3}{2}$.

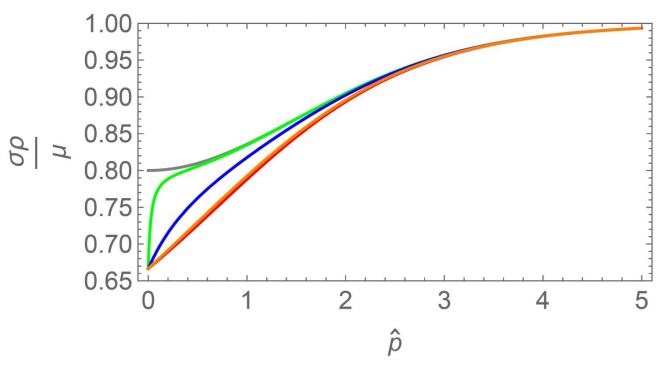

Fig. 12. Densities $\rho$ for $\frac{H}{\sigma} \rightarrow 1^{+}$(black curve) and $\frac{H}{\sigma} \rightarrow\left(1+\frac{\sqrt{3}}{2}\right)^{-}$with $\hat{\omega}=0$ (gray curve), 0.1 (red curve), 0.5 (green curve), 2 (blue curve), $\infty$ (orange curve) are depicted. Curves for $\frac{H}{\sigma} \rightarrow 1^{+}$and $\omega=\infty$ lie on top of each other while results for $\frac{H}{\sigma} \rightarrow(1+$ $\left.\frac{\sqrt{3}}{2}\right)^{-}$are increased in comparison to the density presented in Fig. 6 for $\frac{H}{\sigma}=\frac{3}{2}$.

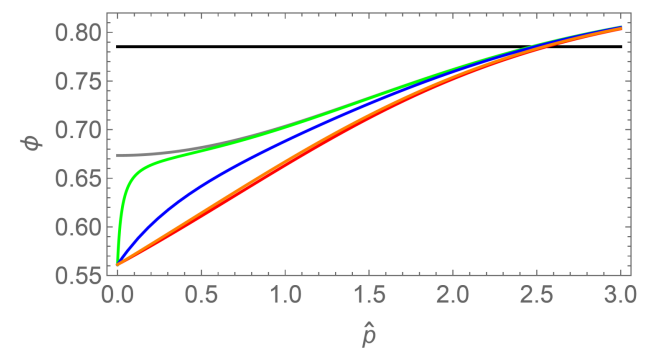

Fig. 13. Packing factors $\phi$ for $\frac{H}{\sigma} \rightarrow 1^{+}$(black curve) and $\frac{H}{\sigma} \rightarrow\left(1+\frac{\sqrt{3}}{2}\right)^{-}$with $\hat{\omega}=0$ (gray curve), 0.1 (red curve), 0.5 (green curve), 2 (blue curve), $\infty$ (orange curve) are depicted.

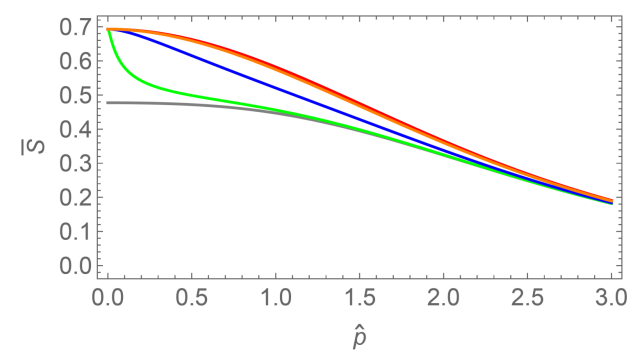

Fig. 14. Entropy $\bar{S}$ for $\frac{H}{\sigma} \rightarrow 1^{+}$(black curve) and $\frac{H}{\sigma} \rightarrow\left(1+\frac{\sqrt{3}}{2}\right)^{-}$with $\hat{\omega}=0$ (gray curve), 0.1 (red curve), 0.5 (green curve), 2 (blue curve), $\infty$ (orange curve) are depicted. Curves for $\frac{H}{\sigma} \rightarrow 1^{+}$and $\omega=\infty$ lie on top of each other.

scaled rotation $\hat{\omega}=\infty$, respectively. The result (46) for the packing factor corresponds to the trivial geometric expectation. Complementary, disks with diameters equal to the container diameter correspond to disks under infinitely large scaled rotations $(\hat{\omega}=\infty)$ since, as expected, the disks are pinned to one side of the tube which resembles stacked disks. Only the packing factor $\phi$ varies as shown in (46). 


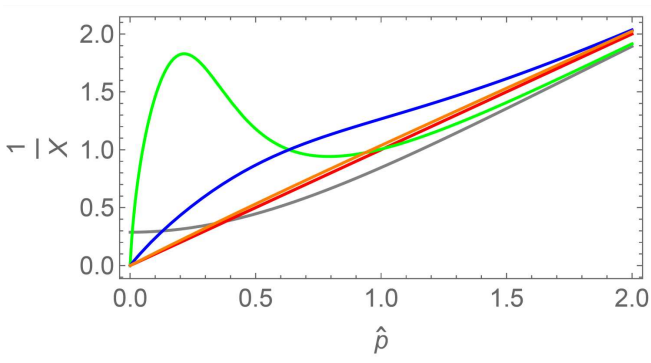

Fig. 15. Reciprocal compactivities $X^{-1}$ for $\frac{H}{\sigma} \rightarrow$ $1^{+}$(black curve) and $\frac{H}{\sigma} \rightarrow\left(1+\frac{\sqrt{3}}{2}\right)^{-}$with $\hat{\omega}=0$ (gray curve), 0.1 (red curve), 0.5 (green curve), 2 (blue curve), $\infty$ (orange curve) are depicted. Curves for $\frac{H}{\sigma} \rightarrow 1^{+}$and $\omega=\infty$ lie on top of each other. The packing factor is constantly $\pi / 4$ for $\frac{H}{\sigma} \rightarrow 1^{+}$.

On the other hand, in the limiting case $\frac{H}{\sigma} \rightarrow$ $\left(1+\frac{\sqrt{3}}{2}\right)^{-}$, one obtains results similar to Figs. $5-9$, depicted in Figs. 11-15. The population correlation $\bar{N}_{1}$ remains unchanged since it does not depend on $\frac{H}{\sigma}$. The value of $\bar{N}_{2}$ decreases for $\frac{H}{\sigma} \rightarrow\left(1+\frac{\sqrt{3}}{2}\right)^{-}$ leading to a decreasing $\bar{V}$ but increasing $\frac{\sigma \rho}{\mu}$. In fact, this is in agreement with the geometric expectation resulting from decreasing disk diameters $\sigma$.

\section{Conclusions}

The framework of this study is based on the generalized Pauli Principle. The statistical mechanics established for quantum many-body systems is used for classical quasiparticles. In this context, it is assumed that the jammed macrostates are generated by uniform random agitations and controllable by some variables, i.e., scaled pressure $\hat{p}$ and rotation $\hat{\omega}$.

The explicit results of this study, obtained rigorously from the structure of the configurational statistics, lead to a situation wherein these results can be examined by use of experiments, simulations and other basic theories. An exemplary comparison of the obtained entropy $\bar{S}$ with the previous results [61] was performed. Especially, the simplicity of the presented analysis (due to the consideration of only flat, rigid, frictionless disks in a narrow tube) combined with the complex results make the model of this study particularly suitable for these purposes.

Apart from this observation, external fields give characteristic profiles for the volume $\bar{V}$, mass density $\rho$, packing factor $\phi$, entropy $\bar{S}$ and compactivity $X$ of the granular matter. In the presented model jamming occurs due to the local pressure $p(z)$ in a uniform gravitational field parallel to the tube in the $z$ direction and rotation $\omega$. The beforementioned properties of the granular matter in addition to the characteristics (population densities $|\bar{N}\rangle$ and population correlations $\left.\rho_{11}, \rho_{12}, \rho_{22}\right)$ of the quasiparticles were determined. Remarkably, this simple framework with just one host and one tag leads to involved results for complex systems which typically show different behaviour for vanishing rotation $\hat{\omega}=0$ in comparison to finite rotations $\hat{\omega}$. Especially the entropy $\bar{S}$ is of great interest. In dependence of the volume $\bar{V}$ it starts at $\bar{S}=0$ with infinite slope and ends at $\ln (2)$ for $\hat{\omega} \neq 0$ while it starts at $\bar{S}=0$ with the finite initial slope $\left[\ln \left(\frac{4}{3}\right)\right]^{-1}$ and ends at $\frac{3}{4} \ln (3)-\frac{1}{2} \ln (2)$ for vanishing rotations $\hat{\omega}=0$. In addition, the entropy $\bar{S}$ exhibits concave behaviour. Accordingly, the compactivity $X$ varies between $X=0$ at $\bar{V}=0$ and $X=\infty$ at $\bar{V}=\frac{1}{2}$ for $\hat{\omega} \neq 0$. Thus, it is a measure for the magnitude with which the system is arbitrarily agitated to yield a distinct jammed macrostate. Application of strong random agitations leads to a highly compressible state with exceedingly large volume $\bar{V}$. In contrast, shaking the matter with a smaller magnitude leads to a more compact and less compressible jammed macrostate.

Complementary to these results, the presented framework should be considered as a first step since by considering the entropy $\bar{S}$ it was shown that even such a simple system that is exactly solvable can fit to results of complex systems. Obviously, it is open to further improvement with other effects like different configurations caused by use of $1+\frac{\sqrt{3}}{2}<$ $\frac{H}{\sigma}<2$ or $\frac{H}{\sigma}>2$ leading to novel sets of quasiparticles, friction, varying axis orientation, masses and radii.

\section{Acknowledgments}

The author acknowledges financial support by the Scientific and Technological Research Council of Turkey within the programs 1515 and 1004 within the projects 5189901 and 20AG001, respectively. Moreover, the author is grateful to the Institute of Materials Science and Nanotechnology at Bilkent University and Faculty of Engineering at Middle East Technical University for providing the needed infrastructure.

\section{References}

[1] R.K. Bowles, Physica A 275, 217 (2000).

[2] H.P. Zhang, H.A. Makse, Phys. Rev. E 72, 011301 (2005).

[3] C. Song, P. Wang, H.A. Makse, Nature 453, 629 (2008).

[4] R. Mari, F. Krzakala, J. Kurchan, Phys. Rev. Lett. 103, 025701 (2009).

[5] M. van Hecke, J. Phys. Condens. Matter 22, 033101 (2009).

[6] G. Parisi, F. Zamponi, Rev. Mod. Phys. 82, 789 (2010). 
[7] M.P. Ciamarra, A. Coniglio, A. de Candia, Soft Matter 6, 2975 (2010).

[8] M.P. Ciamarra, M. Nicodemi, A. Coniglio, Soft Matter 6, 2871 (2010).

[9] R. Dewar, J. Phys. A Math. Gen. 36, 631 (2003).

[10] R.C. Dewar, J. Phys. A Math. Gen. 38, 371 (2005).

[11] S. Alexander, Phys. Rep. 296, 65 (1998).

[12] P.G. de Gennes, Rev. Mod. Phys. 71, S374 (1999).

[13] A. Crisanti, F. Ritort, J. Phys. A Math. Gen. 36, 181 (2003).

[14] J.M. Ottino, Chem. Eng. Sci. 61, 4165 (2006).

[15] S. Torquato, F.H. Stillinger, Rev. Mod. Phys. 82, 2633 (2010).

[16] Y. Jin, P. Charbonneau, S. Meyer, C. Song, F. Zamponi, Phys. Rev. E 82, 051126 (2010).

[17] E. DeGiuli, J. McElwaine, Phys. Rev. E 84, 041310 (2011).

[18] F. Paillusson, D. Frenkel, Phys. Rev. Lett. 109, 208001 (2012).

[19] G.D. Scott, D.M. Kilgour, J. Phys. D: Appl. Phys. 2, 863 (1969).

[20] T. Pöschel, H.J. Herrmann, Europhys. Lett. 29, 123 (1995).

[21] H.A. Makse, D.L. Johnson, L.M. Schwartz, Phys. Rev. Lett. 84, 4160 (2000).

[22] N. Xu, J. Blawzdziewicz, C.S. O'Hern, Phys. Rev. E 71, 061306 (2005).

[23] A. Donev, F.H. Stillinger, S. Torquato, Phys. Rev. Lett. 95, 090604 (2005).

[24] F. Lechenault, F. da Cruz, O. Dauchot, E. Bertin, J. Stat. Mech. 2006, 07009 (2006).

[25] G.J. Gao, J. Bławzdziewicz, C.S. O'Hern, Phys. Rev. E 74, 061304 (2006).

[26] D. Aristoff, C. Radin, J. Stat. Phys. 135, 1 (2009).

[27] M. Danisch, Y. Jin, H.A. Makse, Phys. Rev. E 81, 051303 (2010).

[28] C.F. Schreck, C.S. O'Hern, L.E. Silbert, Phys. Rev. E 84, 011305 (2011).

[29] S. Zivkovic, Z. Jaksic, D. Arsenovic, L. Budinski-Petkovic, S. Vrhovac, Acta Phys. Pol. A 120, 246 (2011).

[30] K.A. Newhall, I. Jorjadze, E. VandenEijnden, J. Brujic, Soft Matter 7, 11518 (2011).

[31] R. Pastore, M.P. Ciamarra, A. Coniglio, Granul. Matter 14, 253 (2012).

[32] R. Hihinashvili, R. Blumenfeld, Granul. Matter 14, 277 (2012).
[33] K.V. Edmond, C.R. Nugent, E.R. Weeks, Phys. Rev. E 85, 041401 (2012).

[34] S. Franz, H. Jacquin, G. Parisi, P. Urbani, F. Zamponi, J. Chem. Phys. 138, 12A540 (2013).

[35] C. Brito, G. Parisi, F. Zamponi, Soft Matter 9, 8540 (2013).

[36] A. Baule, F. Morone, H.J. Hermann, H.A. Makse, Rev. Mod. Phys. 90, 015006 (2018).

[37] A. Lemaitre, C. Mondal, I. Procaccia, S. Roy, Y. Wang, J. Zhang, Phys. Rev. Lett. 126, 075501 (2021).

[38] S.F. Edwards, R.B.S. Oakeshott, Physica A 157, 1080 (1989).

[39] R. Blumenfeld, S.F. Edwards, Eur. Phys. J. E 19, 23 (2006).

[40] S.F. Edwards, J. Phys. A Math. Theor. 41, 324019 (2008).

[41] S. Henkes, B. Chakraborty, Phys. Rev. E 79, 061301 (2009).

[42] L. Berthier, H. Jacquin, F. Zamponi, J. Stat. Mech. 2011, 01004 (2011).

[43] K. Wang, C. Song, P. Wang, H.A. Makse, Phys. Rev. E 86, 011305 (2012).

[44] J.G. Puckett, K.E. Daniels, Phys. Rev Lett. 110, 058001 (2013).

[45] R.P. Behringer, B. Chakraborty, Rep. Prog. Phys. 82, 012601 (2019).

[46] C. Radin, H.L. Swinney, J. Stat. Phys. 175, 542 (2019).

[47] A. Megias, A. Santos, Granul. Matter 21, 49 (2019).

[48] A. Mehta, S.F. Edwards, Physica A 157, 1091 (1989).

[49] B.D. Lubachevsky, F.H. Stillinger, J. Stat. Phys. 60, 561 (1990).

[50] T.S. Majumdar, M. Sperl, S. Luding, R.P. Behringer, Phys. Rev. Lett. 98, 058001 (2007).

[51] C.S. O'Hern, S.A. Langer, A.J. Liu, S.R. Nagel, Phys. Rev. Lett. 88, 075507 (2002).

[52] A.P. Polychronakos, Phys. Lett. B 365, 202 (1996).

[53] W.H. Huang, Phys. Rev. B 53, 15842 (1996).

[54] A.K. Aringazin, M.I. Mazhitov, Phys. Rev. E 66, 026116 (2002).

[55] C. Vitoriano, R.R. Montenegro-Filho, M.D. Coutinho-Filho, Phys. Rev. B 98, 085130 (2018).

[56] N.E. Fahssi, Mod. Phys. Lett. B 33, 1950244 (2019).

[57] W. Pauli, Z. Phys. 31, 765 (1925). 
[58] F.D.M. Haldane, Phys. Rev. Lett. 67, 937 (1991).

[59] Y.S. Wu, Phys. Rev. Lett. 73, 922 (1994).

[60] Y.S. Wu, Phys. Rev. Lett. 104, 198902 (2010).

[61] Y. Jin, H.A. Makse, Physica A 389, 5362 (2010).

[62] Y. Öz, M. Karbach, G. Müller, "Population Correlations of Statistically Interacting Quasiparticles in Ising Spin" (2021), (to be published). 